\title{
Practical Bifurcation Criteria considering Inductive Power Pad Losses in Wireless Power Transfer Systems
}

\author{
Minkook Kim*, Jae-Woo Lee** and Byoung Kuk Lee ${ }^{\dagger}$
}

\begin{abstract}
In this paper, the bifurcation criteria for inductive power transfer (IPT) systems is suggested considering the inductive power pad losses. The bifurcation criteria for series-series (SS) and series-parallel (SP) topologies are derived in terms of the main parameters of the IPT system. For deriving precise criteria, power pad resistance is obtained by copper loss calculation and core loss analysis. Utilizing the suggested criteria, possibility of bifurcation occurrence can be predicted in the design process. In order to verify the proposed criteria, $50 \mathrm{~W}$ IPT laboratory prototype is fabricated and the feasibilities of the switching frequency and $\mathrm{AC}$ load resistance shift to escape from bifurcation are identified.
\end{abstract}

Keywords: Bifurcation criteria, Frequency splitting, Inductive power pad losses, Inductive power transfer, Wireless power transfer

\section{Introduction}

Inductive power transfer (IPT) systems deliver power to the load through electromagnetic induction without any physical contact. IPT systems have been globally used for various applications such as electric vehicles, mobile devices, etc. In conventional IPT systems, electric source supplies the complex power when the system is not compensated. Thus, compensation capacitors are inserted to minimize the VA rating. According to the connection configuration of compensated capacitors and power pad coils, four basic topologies are derived and among them, series-series (SS) and series-parallel (SP) topologies are widely used due to simplicity of the circuit and constant output characteristic [1]. In order to improve the overall system efficiency, both switching technique and inductive power pad design should be considered. In case of the switching technique, it is necessary for the IPT system to operate in the inductive region for zero voltage switching (ZVS) in order to reduce the switching losses. With the respect of inductive power pads, the coupling coefficient $k$ and the quality factors $Q$ should be considered [2]. Several studies on magnetic coupling proved that high $k$ plays a critical role in improving the efficiency [3]. However, if the designed $k$ exceeds certain limit in the IPT system, single resonant frequency is split into three resonant frequencies, even, odd, and initially designed

$\uparrow$ Corresponding Author: Department of Electrical and Computer Engineering, Sungkyunkwan University, Korea. (bkleeskku@skku.edu)

* Department of Electrical and Computer Engineering, Sungkyunkwan University, Korea. (mkfour44@skku.edu)

** Living \& Energy R\&D center, LG Electronics, Korea. (jaewoo11.lee@lge.com)

Received: May 27, 2016; Accepted: July 18, 2016 frequencies [4]. This phenomenon is defined as frequency splitting or bifurcation. Bifurcation is an especially serious problem in the IPT systems because the variation of system characteristics due to bifurcation directly affects the system reliability and stable operation range [5]. If bifurcation occurs by high coupling coefficient, caused by misalignment of secondary power pad, the difference of inherent resonant frequencies, $\omega_{\text {even }}$ and $\omega_{\text {odd }}$, is expanded. This resonant frequency variation could change the operation point from ZVS to zero current switching (ZCS), resulting in higher switching losses. The ideal parameters of the resonant network in IPT systems are composed of inductance and capacitance. However, in the practical systems, loss components of inductive power pads exist, which can be represented as equivalent resistance. Consequently, this equivalent resistance leads to reactive component in reflected impedance on the primary power pad even though the secondary power pad is compensated.

Previous research on bifurcation concentrated on the variations of voltage gain, impedance, and phase under bifurcation occurrence [6]. The other study introduced the bifurcation criteria based on numerical analysis [1]. However, both researches did not consider the effects of the inductive power pad losses and till now, practical bifurcation criteria for IPT system is not suggested.

Therefore, in this paper, the bifurcation criteria that takes into account the inductive power pad losses are derived and they are categorized on the basis of SS and SP topologies. The inductive power pad resistances are calculated through the analysis of copper and ferrite core losses. The proposed criteria are theoretically explained in detail and the validity of the proposed criteria is verified by simulation and experimental results. 


\section{Bifurcation Phenomena in IPT Systems}

\subsection{Definition}

The typical IPT system consists of inductive power pads, compensation capacitors, and the equivalent load. In order to identify bifurcation in the IPT system, input impedance $Z_{\text {in }}$ influenced by magnetic coupling has to be analyzed. Fig. 1 shows an impedance schematic of the primary series compensated IPT system. In this system, the lumped secondary impedance $Z_{22}$ is defined as follows.

$$
\begin{aligned}
Z_{22} & =\operatorname{Re}\left[Z_{22}\right]+j \operatorname{Im}\left[Z_{22}\right] \\
& =j \omega_{s} L_{2}+\frac{1}{j \omega_{s} C_{2}}+R_{e q} \quad \text { series compensated } \\
& =j \omega_{s} L_{2}+\frac{R_{e q}}{1+j \omega_{s} C_{2} R_{e q}} \quad \text { parallel compensated }
\end{aligned}
$$

where, $L_{2}$ is secondary pad inductance, $C_{2}$ is secondary compensation capacitor, $\omega_{s}$ is switching angular frequency of the IPT system, and $R_{e q}$ is equivalent ac load resistance [7]. The reflected impedance $Z_{r}$ is given by

$$
Z_{r}=\frac{\left(\omega_{s} M\right)^{2}}{Z_{22}}=\left(\frac{\omega_{s} M}{Z_{22}}\right)^{2} \times\left(\operatorname{Re}\left[Z_{22}\right]-j \operatorname{Im}\left[Z_{22}\right]\right)
$$

where, $M$ is the mutual inductance between primary and secondary power pads and is expressed as $k\left(L_{1} L_{2}\right)^{1 / 2}$. From Fig. 1, the input impedance $Z_{i n}$ is expressed as

$$
Z_{\text {in }}=Z_{1}+Z_{r}
$$

where, $Z_{l}$ is combined impedance of $L_{l}$ and $C_{l}$. It is proved

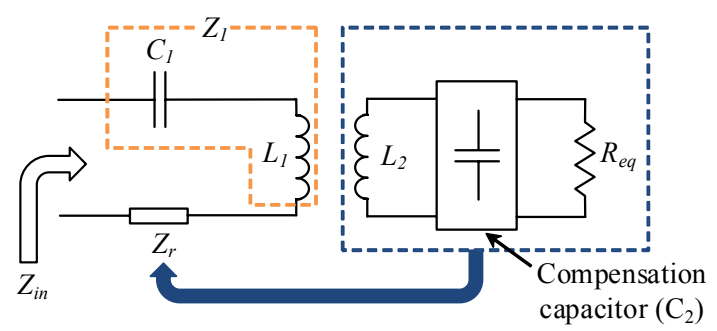

Fig. 1. Impedance schematic of the primary series compensated IPT system

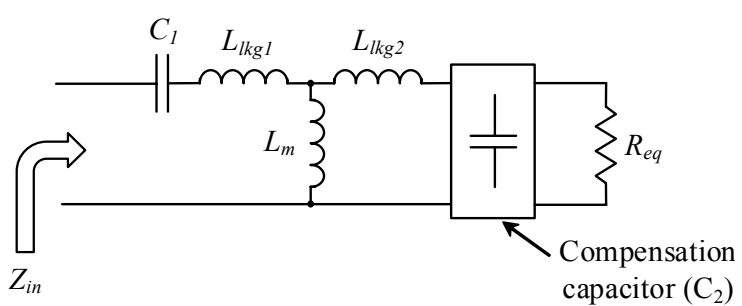

Fig. 2. T-model of the input impedance $Z_{\text {in }}$ that the resonance of the IPT systems occurs at three points through the T-model analysis of input impedance as shown in Fig. 2. These three points are defined as initially designed resonant frequency $\omega_{0}$, odd frequency $\omega_{\text {odd }}$, and even frequency $\omega_{\text {even }}$. $\omega_{\text {odd }}$ and $\omega_{\text {even }}$ are affected by the magnetizing inductance $L_{m}$, which is obtained by applying the coupled inductor model and the T-model $[4,8]$.

$$
L_{m}=k \sqrt{L_{1} L_{2}} \times \frac{N_{1}}{N_{2}}
$$

where, $N_{1}$ and $N_{2}$ are the number of equivalent turns of the primary and secondary power pads. From (4), the value of $L_{m}$ decreases when the magnetic coupling of the IPT system is weak and in that case, $\omega_{\text {odd }}$ and $\omega_{\text {even }}$ are approximately identical to $\omega_{0}$. However, as shown in Fig. 3 , the difference between $\omega_{\text {odd }}$ and $\omega_{\text {even }}$ is expanded as $k$ increases. This phenomenon is called bifurcation in the IPT system [9].

\subsection{Problem analysis of bifurcation}

If $\omega_{s}$ is higher than the initially designed resonant frequency $\omega_{0}$, the reactance of the lumped secondary impedance $\operatorname{Im}\left[Z_{22}\right]$ is positive. In that case, the reactive component of $Z_{r}$ is negative from (2). Considering (3), the input reactance $X_{i n}$ is the reactive component sum of $Z_{1}$ and $Z_{r}$. Thus, in the case of weak magnetic coupling IPT system, operation region of the system is not changed, so $Z_{\text {in }}$ is inductive. However, if $k$ is higher than the critical point,

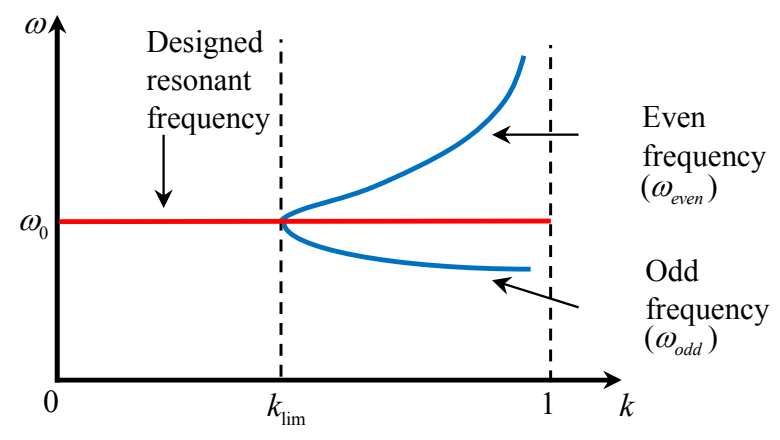

Fig. 3. Resonant frequency splitting by bifurcation

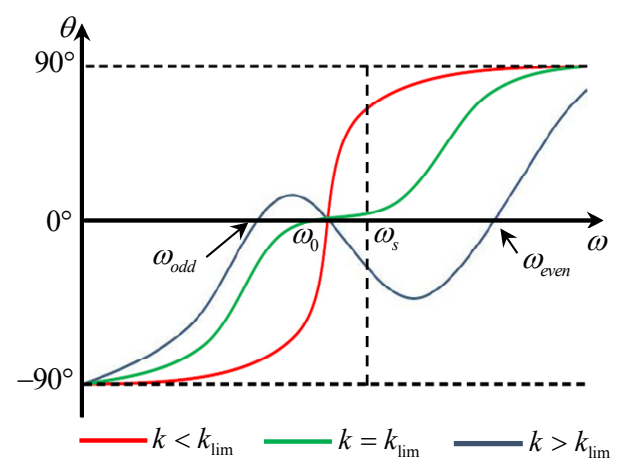

Fig. 4. Phase curve variation of input impedance with coupling coefficient 
defined as $k_{\text {lim }}$, bifurcation occurs and the polarity of the operation region is changed into capacitive. In that case, $\omega_{s}$ is lower than the split frequency $\omega_{\text {even }}$ as shown in Fig. 4. This reactive component variation can change the operating point of the IPT system from the ZVS region to the ZCS region. Therefore, the sudden phase variation in the operating region due to bifurcation causes system instability. Also, the voltage curve variation causes undesirable effect on system stability. The voltage gain $G_{v}$ of the SS and SP topologies are as follows [10].

$$
\begin{array}{rlr}
G_{v} & =v_{\text {out }} / v_{\text {in }} & \\
& =\left|\frac{j \omega_{s} M}{Z_{\text {in }}}\right| \times\left|\frac{R_{\text {eq }}}{Z_{22}}\right| & \text { (SS topology) } \\
& =\left|\frac{j \omega_{s} M}{Z_{\text {in }}}\right| \times\left|\frac{1}{Z_{22}}\right| \times\left|\frac{R_{\text {eq }}}{1+j \omega_{s} C_{2} R_{\text {eq }}}\right| & \text { (SP topology) }
\end{array}
$$

Figs. 5 and 6 show the voltage gain curves of the SS and SP topologies. In general, the $G_{v}$ of the primary series compensated topologies has only one peak if the IPT system is operated at $k<k_{\lim }$ because of the $M^{2}$ component in $Z_{\text {in }}$. However, at $k>k_{\text {lim }}, G_{v}$ is boosted at the frequencies of $\omega_{\text {odd }}$ and $\omega_{\text {even }}$. This double-peak characteristic of the

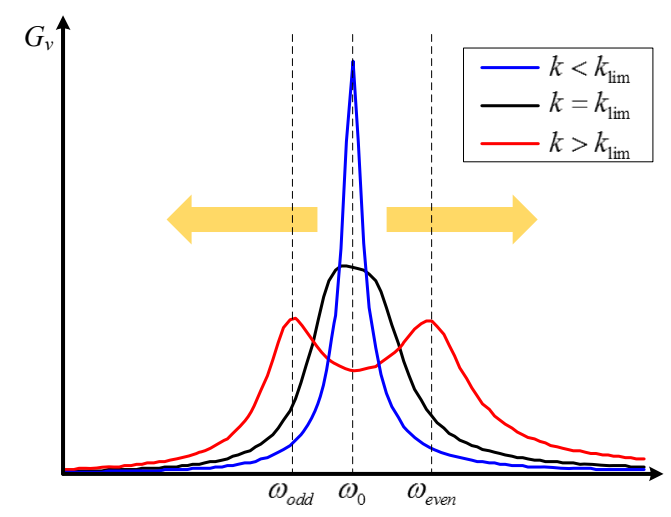

Fig. 5. Voltage gain of SS topology with the coupling coefficient and frequency variation

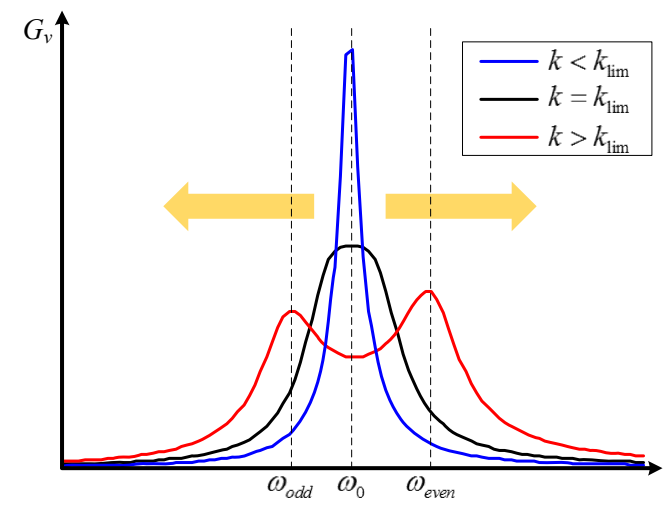

Fig. 6. Voltage gain of SP topology with the coupling coefficient and frequency variation voltage gain is caused by $Z_{r}$ variation. Especially the $G_{v}$ at $\omega_{\text {even }}$ of SP topology is relatively higher than that of the SS topology. This difference is caused by $1 /\left(1+j \omega_{\mathrm{s}} C_{2} R_{\text {eq }}\right)$ term in the SP topology [10]. The difference between $\omega_{\text {odd }}$ and $\omega_{\text {even }}$ in the IPT system is more expanded as $k$ increases.

As explained above, the IPT system shows complex characteristics in the bifurcation region. Therefore, in the system design process, bifurcation has to be taken into account for system stability. In this respect, the bifurcation criteria derivation is important to operate the IPT system in the bifurcation free region. In order to derive the exact criteria, two things should be considered. One is the inductive power pad losses which can be substituted by equivalent resistance. The equivalent resistance changes the both magnitude and phase angle of $Z_{i n}$. Thus, the equivalent pad resistance calculation has to be included in the criteria derivation process. The other consideration is different $Z_{\text {in }}$ expressions depending on the topologies of the IPT system.

\section{Bifurcation Criteria}

\subsection{Inductive power pad losses}

\subsubsection{Copper loss}

The AC copper losses of coil windings are calculated by using the $\mathrm{AC}$ resistance of the Litz wire and the current value. By utilizing the Litz wire, proximity and skin effect caused by high frequency $\mathrm{AC}$ currents can be minimized. However, owing to the spiral coil structure of the inductive power pads, the proximity effect caused by the external magnetic intensity $H_{e}$ has to be analyzed to derive the precise copper losses. Fig. 7 shows the cross-sectional area of the Litz wire winding. Litz wire winding consists of $n$ strands, and the diameter of each strand is designated as $t_{i}$. In order to derive the skin effect loss, Maxwell's equation and ohmic law under the cylindrical symmetric condition of a round conductor is used [11]. For a sinusoidal current $i(t)=I_{\text {peak }} \sin \left(\omega_{s} t\right)$ passing through the circular spiral winding, the total skin effect loss is calculated as



Fig. 7. Cross-sectional area of Litz wire winding 


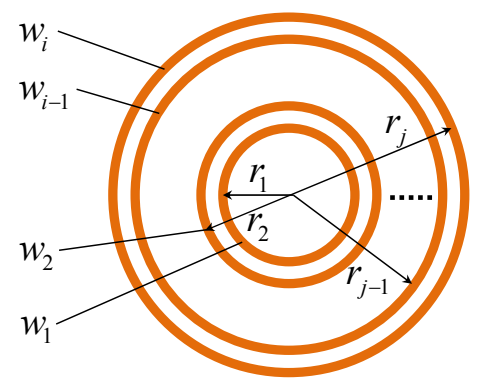

Fig. 8. Equivalent circular spiral winding: the order of turn $w_{i}$ and the coil radius $r_{j}$

$$
P_{\text {skin }}=n \cdot R_{D C} \cdot F_{R}\left(f_{S}\right) \cdot\left(\frac{I_{p e a k}}{n}\right)^{2} \times l_{\text {coil }}
$$

where, $R_{D C}$ is DC resistance per unit length of a single strand of the Litz wire, $F_{R}\left(f_{s}\right)$ is a skin effect factor that describes the increasing rate in the single-strand conductor resistance due to skin effect, and $l_{\text {coil }}$ is the total length of copper [12]. The proximity effect loss is induced by the $H_{e}$ and the internal magnetic intensity $H_{i}$. The $H_{i}$ across the strand is generated from lateral strands and $H_{e}$ originates from the lateral Litz wire [13]. The total proximity effect loss caused by $H_{i}$ and $H_{e}$ is calculated as

$$
\begin{aligned}
P_{\text {prox }} & =P\left(H_{i}\right)+P\left(H_{e}\right) \\
& =\sum_{j=1}^{N}\left[n \cdot R_{D C} \cdot G_{R}\left(f_{S}\right) \cdot\left(\hat{H}_{e}^{2}\left(w_{i}\right)+\frac{I_{\text {peak }}^{2}}{2 \pi^{2} d^{2}}\right)\right] \times 2 \pi r_{j}
\end{aligned}
$$

where, $G_{R}\left(f_{s}\right)$ is a proximity effect factor expressed in terms of the Kelvin function, and $r_{j}$ is the radius of the equivalent circular spiral winding as shown in Fig. 8 [14]. The sum of the external magnetic intensities $H_{e}\left(w_{i}\right)$ across one winding $w_{i}$ is calculated as (8) and the total resistance $R_{\text {copper }}$ due to the copper loss is expressed as (9).

$$
\begin{gathered}
\hat{H}_{e}\left(w_{i}\right)=\sum_{j=1}^{N} \hat{H}\left(r_{j}\right)(j \neq i) \\
R_{\text {copper }}=2 \times\left(P_{\text {skin }}+P_{\text {prox }}\right) / I_{\text {peak }}^{2}
\end{gathered}
$$

\subsubsection{Core loss}

The ferrite core loss $P_{\text {core }}$ can be estimated by integrating the core loss density $p_{\text {core }}\left[\mathrm{W} / \mathrm{m}^{3}\right]$ according to the Steinmetz equation [15].

$$
\begin{gathered}
p_{\text {core }}=\kappa \times f_{S}^{\alpha} \times B_{\text {peak }}^{\beta} \\
P_{\text {core }}=\int_{v} p_{\text {core }} d v=P_{\text {joule }}+P_{\text {hysteresis }}
\end{gathered}
$$

The Steinmetz coefficients are derived from the B-P curve. In this analysis, a TDK PC95 ferrite core is used.
The coefficients are $\kappa=11.35, \alpha=1.234, \beta=2.322$ under the following condition: $25^{\circ} \mathrm{C}, f_{s}=100 \mathrm{kHz}$ [16]. The magnetic flux density is simulated by finite element method (FEM) simulation. The additional resistance $R_{\text {core }}$ due to the core loss is expressed as

$$
R_{\text {core }}=2 \times P_{\text {core }} / I_{\text {peak }}^{2}
$$

In order to obtain the core loss, a current source is connected to one pad while the other pad is open-circuited in the FEM simulation. The equivalent resistances of each pad is calculated as

$$
\begin{aligned}
& R_{1}=R_{\text {copper }(\text { pri })}+R_{\text {core }(\text { pri })}+R_{\text {core }(\text { sec_open })} \\
& R_{2}=R_{\text {copper }(\mathrm{sec})}+R_{\text {core }(\mathrm{sec})}+R_{\text {core }(\text { pri_open })} \\
& X_{\text {in_s }}=\omega L_{1}-\frac{1}{\omega C_{1}}-\frac{\omega^{2} M^{2}\left(\omega L_{2}-\frac{1}{\omega C_{2}}\right)}{\left(R_{2}+R_{e q}\right)^{2}+\left(\omega L_{2}-\frac{1}{\omega C_{2}}\right)^{2}} \\
& X_{\text {in_sP }}=\omega L_{1}-\frac{1}{\omega C_{1}} \\
& \quad-\frac{\omega^{2} M^{2}\left(\omega L_{2}\left(\left(\omega C_{2} R_{e q}\right)^{2}+1\right)-\omega C_{2} R_{e q}^{2}\right)}{\left(\left(\omega C_{2} R_{e q}\right)^{2}+1\right)\left(R_{2}^{2}+\left(\omega L_{2}\right)^{2}\right)+2 R_{e q}\left(R_{2}-\omega^{2} L_{2} C_{2} R_{e q}\right)+R_{e q}^{2}}
\end{aligned}
$$

\subsection{Criteria of SS and SP topologies}

The bifurcation criteria $k_{\text {lim }}$ and the coupling coefficient $k_{\text {even }}$ for the case that $\omega_{s}$ equals $\omega_{\text {even }}$ can be derived with $X_{\text {in }}$ analysis. The $X_{i n}$ values of the SS and SP topologies are given by (15) and (16). In order to simplify the analysis, $X_{\text {in }}$ has to be converted to the main factors such as the quality factor of pad $Q$ at $\omega_{0}$, normalized frequency $\omega_{n}$, and the resistance ratio $R_{n}$. The main factors are defined as follows.

$$
\begin{gathered}
Q_{2}=\omega_{0} L_{2} / R_{2} \\
\omega_{n}=\omega_{S} / \omega_{0} \\
R_{n}=R_{2} / R_{e q}
\end{gathered}
$$

In the SS topology, the compensation capacitors are

$$
\begin{aligned}
& C_{1}=\frac{1}{\omega_{0}^{2} L_{1}} \\
& C_{2}=\frac{1}{\omega_{0}^{2} L_{2}}
\end{aligned}
$$

The basic idea for the derivation of $k_{\text {lim }}$ and $k_{\text {even }}$ is that $X_{\text {in }}$ equals to zero at the designed resonant angular frequency $\omega_{0}$ and the switching frequency $\omega_{s}$. In particular, limit concept is needed to obtain the criteria because $X_{\text {in }}$ is 
zero in all the couplings at $\omega_{0}$. Using (15) and (17)-(21), the $k_{\text {even }}$ of the SS topology is expressed as

$$
k_{\text {even }}=\sqrt{\left(\frac{1}{\omega_{n} Q_{2}}\right)^{2}\left(1+\frac{1}{R_{n}}\right)^{2}+\left(1-\frac{1}{\omega_{n}^{2}}\right)^{2}}
$$

The bifurcation criterion of the SS topology is simplified to

$$
k_{\mathrm{SS} \_\lim }=\lim _{\omega_{n} \rightarrow 1+} k_{S S}=\frac{R_{2}+R_{e q}}{\omega L_{2}}
$$

The derivation of $k_{\lim }$ and the $k_{\text {even }}$ for the SP topologies is identical to that of the SS topology. However, the capacitor design of the SP topologies is classified as the primary and secondary tuning methods. The first method is the $C_{2}$ tuning method. $C_{2}$ is designed to resonate with the secondary inductance $L_{2}$ at $\omega_{0}$. Using $C_{2}$ tuning method, $C_{1}$ and $C_{2}$ are determined by (24) and (25).

$$
\begin{gathered}
C_{1}=\frac{1}{\omega_{0}^{2} L_{1}\left(1-k^{2}\right)} \\
C_{2}=\frac{1}{\omega_{0}^{2} L_{2}}
\end{gathered}
$$

Using (16), the $k_{\text {even }}$ of the $C_{2}$ tuned SP topology is expressed as (26) and the bifurcation criterion is simplified as follows.

$$
k_{\mathrm{SP} 2 \_ \text {lim }}=\lim _{\omega_{n} \rightarrow 1+} k_{S P}=\sqrt{1-\left(\frac{Q_{2}^{2}-1}{\left(Q_{2}^{4} R_{n}^{2}+Q_{2}^{2}\left(R_{n}+1\right)^{2}\right)}\right)}
$$

The second method is the $C_{1}$ tuning method [6]. $C_{1}$ is designed to resonate with the primary inductance $L_{1}$ at $\omega_{0}$. $C_{1}$ and $C_{2}$ are determined by (28) and (29).

$$
\begin{aligned}
& C_{1}=\frac{1}{\omega_{0}^{2} L_{1}} \\
& C_{2}=\frac{1}{2 \omega_{0}{ }^{2} L_{2}}\left(1+\sqrt{1-4\left(\frac{\omega_{0} L_{2}}{R_{e q}}\right)^{2}}\right) \\
& k_{\text {even } \mathrm{C}_{2}}=\sqrt{1+\left(\left(R_{n}+1\right)^{2}+\omega_{n}^{2}\left(\frac{1}{Q_{2}^{2}}-1\right)\right) /\left(\left(\omega_{n}^{4}-\frac{1}{Q_{2}^{2}}\right)+\left(\omega_{n}^{2}-1\right)\left(Q_{2}^{2} R_{n}^{2}-2\right)-\frac{1}{\omega_{n}^{2}}\left(R_{n}+1\right)^{2}\right)} \\
& k_{\text {even_ } \mathrm{C}_{1}}=\sqrt{\left(1-\frac{1}{\omega_{n}^{2}}\right)\left(1+\left(\frac{\omega_{n}^{2}}{2}\left(\frac{1}{Q_{2}^{2}}-1\right)\left(1+\sqrt{1-4 Q_{2}^{2} R_{n}^{2}}\right)-\omega_{n}^{2} R_{n}^{2}+\left(R_{n}+1\right)^{2}\right) / \frac{\left(\omega_{n}^{4}-\omega_{n}^{2}\right)}{2}\left(-2 Q_{2}^{2} R_{n}^{2}+\left(1+\sqrt{1-4 Q_{2}^{2} R_{n}^{2}}\right)\right)\right)}
\end{aligned}
$$

The $k_{\text {even }}$ of the $C_{1}$ tuned SP topology is expressed as (30) and the bifurcation criterion is

$$
k_{S P 1 \_\lim }=\sqrt{\left(\frac{\left(\frac{1}{Q_{2}^{2}}-1\right)\left(1+\sqrt{1-4 Q_{2}^{2} R_{n}^{2}}\right)+4 R_{n}+2}{\left(-2 Q_{2}^{2} R_{n}{ }^{2}+\left(1+\sqrt{1-4 Q_{2}^{2} R_{n}^{2}}\right)\right)}\right)}
$$

\section{Simulation and Experimental Verification}

\subsection{Experimental setup}

The simulation modeling of the inductive power pads is shown in Fig. 9 and the parameters for the simulation are given in Table 1. In order to verify the derived bifurcation criteria, $50 \mathrm{~W}$ laboratory prototype is designed based on the simulation results. The designed $f_{0}$ is $85 \mathrm{kHz}$ and input voltage $V_{s}$ is $55 \mathrm{~V}$. The primary and secondary pad inductances obtained from the simulation are $163.98 \mu \mathrm{H}$ and $20.36 \mu \mathrm{H}$ under the conditions of Table 1 and measured inductances for each pad is $165.23 \mu \mathrm{H}$ and 19.76 $\mu \mathrm{H}$, respectively. In order to apply coil resistance, loss calculation method introduced in Section 3 is employed. The Litz wire parameters are $n=100, t_{i}=0.25 \mathrm{~mm}$, and $d=2.5 \mathrm{~mm}$. The ferrite core material is TDK PC95. The calculated pad resistances are given in Table 2 . The total

Table 1. Parameters of the inductive power pads

\begin{tabular}{c|c}
\hline Parameter & Value [unit] \\
\hline \hline$N_{1}$ & $22[$ turn] \\
\hline$N_{2}$ & $11[$ turn] \\
\hline$r_{\mathrm{i} \text { pri }}$ & $58[\mathrm{~mm}]$ \\
\hline$r_{\mathrm{o} \text { pri }}$ & $150[\mathrm{~mm}]$ \\
\hline$r_{\mathrm{i} \text { sec }}$ & $28[\mathrm{~mm}]$ \\
\hline$r_{\mathrm{o} \text { sec }}$ & $78[\mathrm{~mm}]$ \\
\hline
\end{tabular}

Table 2. Resistances of the inductive power pads

\begin{tabular}{c|c}
\hline Parameter & Value [unit] \\
\hline \hline$R_{\text {skin(pri) }}$ & $83.5[\mathrm{~m} \Omega]$ \\
\hline$R_{\text {prox(pri) }}$ & $51.1[\mathrm{~m} \Omega]$ \\
\hline$R_{\text {core(pri) }}+R_{\text {core(sec_open) }}$ & $31.15[\mathrm{~m} \Omega]$ \\
\hline$R_{\text {skin }(\mathrm{sec})}$ & $23.3[\mathrm{~m} \Omega]$ \\
\hline$R_{\text {prox(sec) }}$ & $11.8[\mathrm{~m} \Omega]$ \\
\hline$R_{\text {core(sec) }}+R_{\text {core(pri_open) }}$ & $1.57[\mathrm{~m} \Omega]$ \\
\hline
\end{tabular}




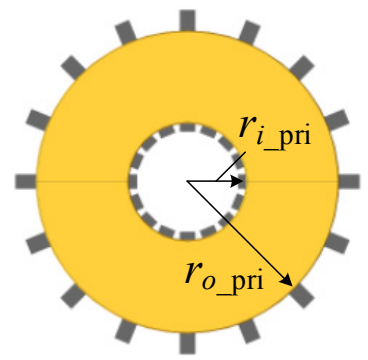

(a) primary pad

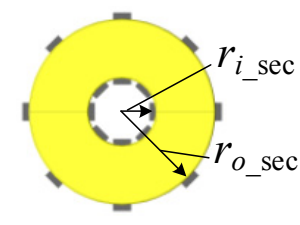

(b) secondary pad
Fig. 9. Modeling of the inductive power pads

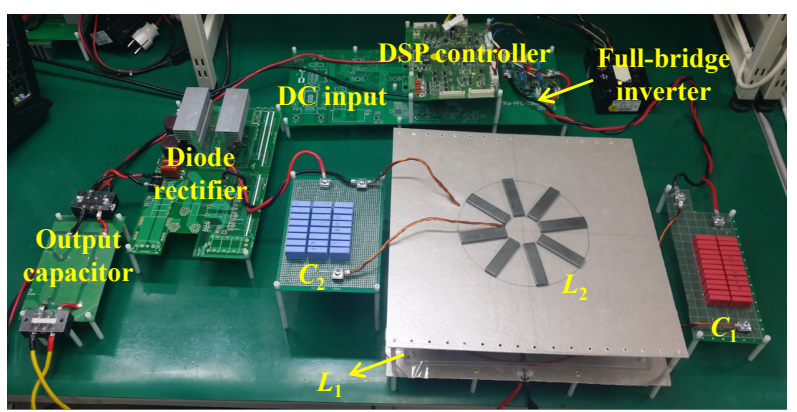

Fig. 10. Laboratory prototype of the IPT system

pad resistance of primary and secondary pad is $165.75 \mathrm{~m} \Omega$, and $36.67 \mathrm{~m} \Omega$, respectively. Using these resistances, the bifurcation criteria of the IPT system are derived using (23), (27) and (31). The compensation capacitors for three types of topologies, SS, $C_{2}$ tuned SP and $C_{1}$ tuned SP topologies, are designed according to the tuning method. Regardless of the compensation network, the values of capacitors are identical and the capacitances of $\mathrm{C}_{1}$ and $\mathrm{C}_{2}$ are $21.36 \mathrm{nF}$, and $169.21 \mathrm{nF}$, respectively. Entire system is illustrated as shown in Fig. 10.

\subsection{Experimental results}

The derived bifurcation criteria and zero phase angle (ZPA) boundary defined by $k_{\text {even }}$ are verified by parameter variation. Initial operating points of three experiments are under bifurcation conditions (point 1, 3, and 5). The operation points of the system are selected based on the bifurcation criteria and ZPA boundary plotting curve. The feasibilities of switching frequency and equivalent AC load resistance variations are tested to escape from bifurcation region.

The bifurcation criterion of the SS topology is shown in Fig. 11. The derived bifurcation criterion $k_{\mathrm{SS} \_ \text {lim }}$ is 0.3 through (23) and the $k$ and $R_{n}$ of this system are 0.4 and 0.0044 , respectively (point 1 ). Through the simulation, it is identified that bifurcation occurs at point 1 and the system is operated under ZCS region. Considering the ZPA boundary curve of Fig. 11, the even frequency $\left(f_{\text {even }}\right)$ of this system at $k=0.4$ is $102 \mathrm{kHz}$. Thus, if $\omega_{\mathrm{s}}$ is shifted to $\omega_{\text {even }}$ (point 2), the IPT system is operated under the ZPA

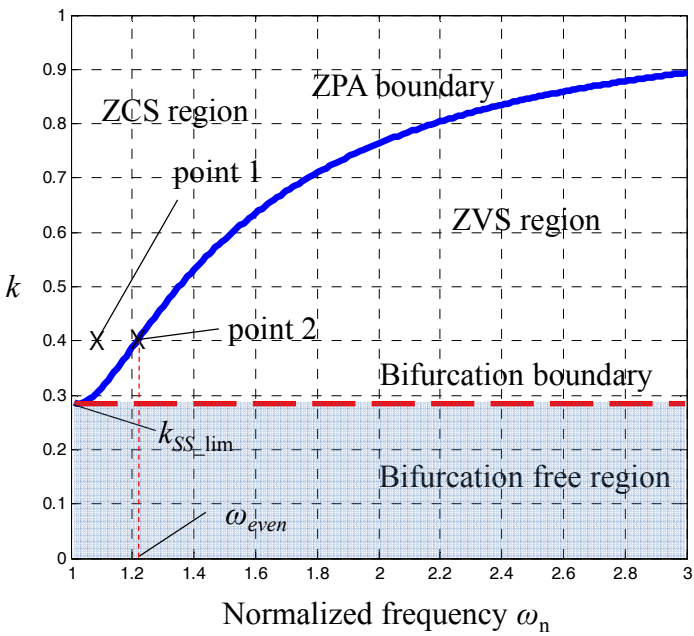

Fig. 11. Bifurcation condition of SS topology. $\left(R_{n}=0.0044\right)$

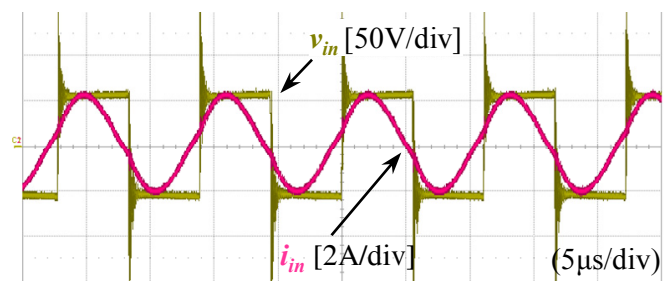

(a) $v_{\text {in }}$ and $i_{\text {in }}$ at point 1

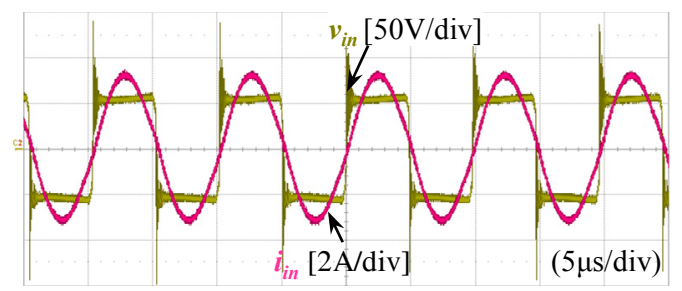

(b) $v_{\text {in }}$ and $i_{\text {in }}$ at point 2

Fig. 12. Experimental waveforms of SS topology

boundary. These analyses are verified by the experimental waveforms at point 1 and 2 presented in Fig. 12.

The bifurcation criterion of the $C_{2}$ tuned SP topology is shown in Fig. 13. The derived bifurcation criterion $k_{\mathrm{SP} 2} \lim$ is 0.092 through (27) and the $k$ and $R_{n}$ of this system are 0.21 and 0.00094 , respectively (point 3 ). At point 3 , the system is operated under both bifurcation and ZCS region. Considering the ZPA boundary curve in Fig. 13, $f_{\text {even }}$ of this system at $k=0.21$ is $93.5 \mathrm{kHz}$. In order to operate the IPT system in the ZVS region, the switching frequency $f_{\mathrm{s}}$ is shifted to $100 \mathrm{kHz}$ (point 4). Experimental waveforms of the input voltage $v_{\text {in }}$ and the input current $i_{\text {in }}$, are shown in Fig. 14. Also, Fig. 13 shows the operation mode conversion from $\mathrm{ZCS}$ region to ZVS region. Although the ZCS mode is changed to the either ZVS mode or ZPA operation, the operation point of the IPT system is still located in the bifurcation region. From the experimental results, it is identified that the switching frequency shift cannot 


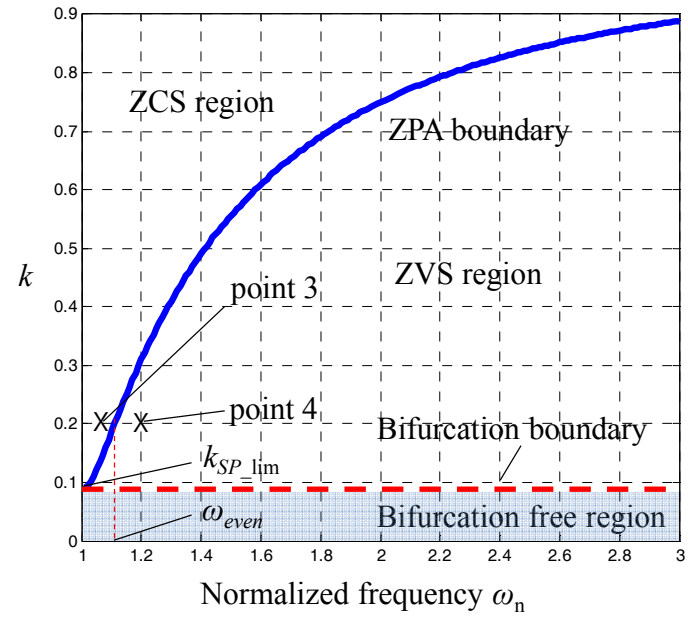

Fig. 13. Bifurcation condition of $C_{2}$ tuned SP topology. $\left(R_{n}=0.00094\right)$

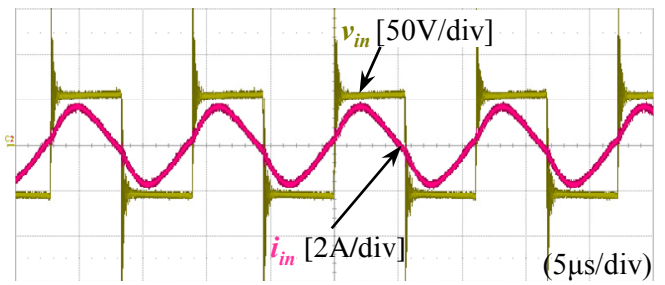

(a) $v_{\text {in }}$ and $i_{\text {in }}$ at point 3

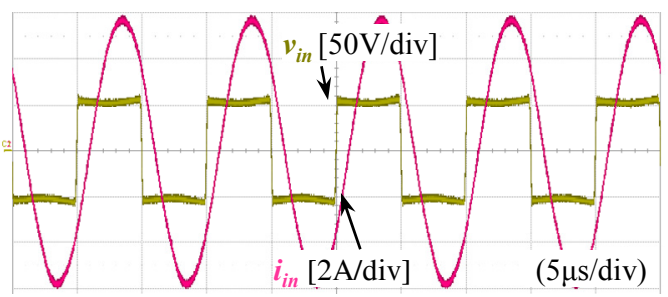

(b) $v_{i n}$ and $i_{i n}$ at point 4

Fig. 14. Experimental waveforms of $C_{2}$ tuned SP topology

eliminate the bifurcation. In order to eliminate bifurcation, the system parameters except $f_{\mathrm{s}}$ have to be changed.

Fig. 15 shows the bifurcation boundary when the equivalent load resistance $R_{e q}$ varies. As the $R_{e q}$ decreases, the bifurcation criterion of the $C_{1}$ tuned SP topology increases. The derived bifurcation criterion $k_{\mathrm{SP} 1} \lim$ is 0.081 through (31). The $k, \omega_{n}$, and $R_{e q}$ of this system are 0.18 , 1.06 and $40.74 \Omega$, respectively (point 5 ). At point 5 , the system is operated under ZCS region. In order to verify the effectiveness of the $R_{n}$ variation, the operating point is changed from 5 to 6 by decreasing the value of the $R_{e q}$ $(=18.34 \Omega)$. The $v_{\text {in }}$ and $i_{\text {in }}$ waveforms at these points are shown in Fig. 16. From this result, it is proved that the $R_{\text {eq }}$ is a crucial parameter to escape from bifurcation. Meanwhile, the ZCS region expressed by the grid area is formed in the free bifurcation region because $f_{0}$ is shifted by the $R_{\text {eq }}$ variation in the $C_{1}$ tuned SP topology system.

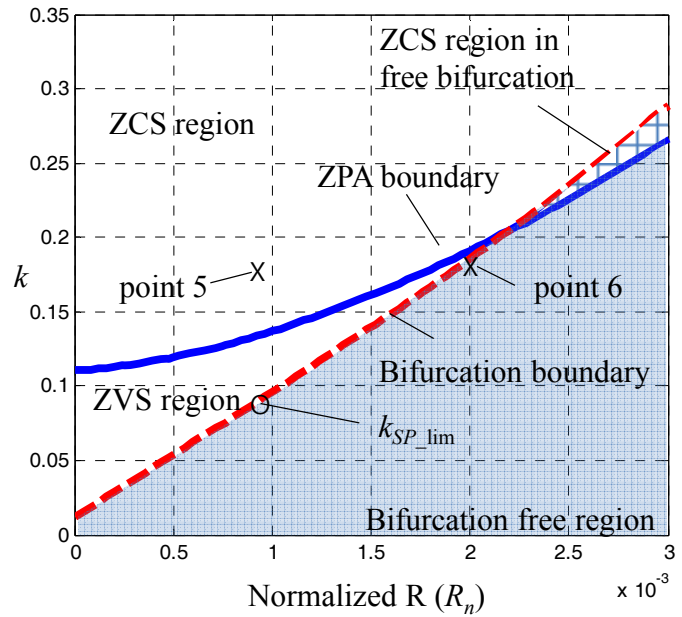

Fig. 15. Bifurcation condition of $C_{1}$ tuned SP topology. $\left(\omega_{n}=1.06\right)$

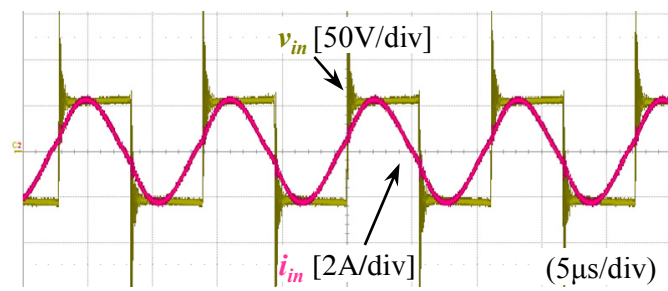

(a) $v_{\text {in }}$ and $i_{\text {in }}$ at point 5

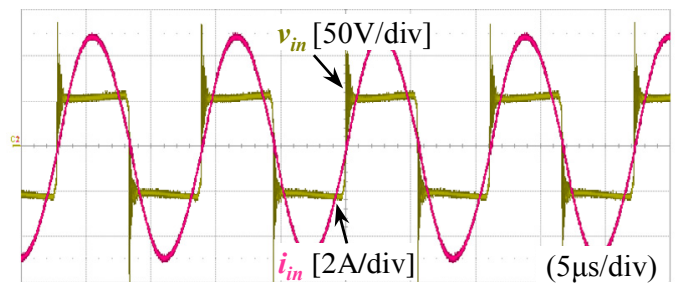

(b) $v_{i n}$ and $i_{\text {in }}$ at point 6

Fig. 16. Experimental waveforms of $C_{1}$ tuned SP topology

Although the system is designed for operation in the ZVS mode, the $R_{e q}$ variation would change the operation mode from ZVS to ZCS mode in free bifurcation region.

\section{Conclusion}

This paper presents practical bifurcation criteria of the SS and SP topologies in the IPT systems. For exact analysis, the inductive power pad losses are considered. For simplicity, main parameters are defined and bifurcation criteria for each topology are expressed based on coupling coefficient. Utilizing derived bifurcation criteria and ZPA boundary, the operation region of the system is categorized into three sections, free bifurcation region, ZVS region, and ZCS region. In order to verify the validity of the proposed criteria, switching frequency and AC load 
resistance shift methods are tested. The switching frequency shift can change not bifurcation region but operation region. However, the AC load resistance shift can make the operation region be displaced from ZCS region to either ZVS region or ZPA boundary and escape from the bifurcation region, simultaneously. Finally, 50 $\mathrm{W}$ laboratory prototype is fabricated and the validity of the analysis is identified.

\section{Acknowledgements}

This work was supported by "Human Resources Program in Energy Technology" of the Korea Institute of Energy Technology Evaluation and Planning (KETEP), granted financial resource from the Ministry of Trade, Industry \& Energy, Republic of Korea. (No.2016 4030200980)

\section{References}

[1] Chwei-Sen Wang, Grant A. Covic, and Oskar H. Stielau, "Power Transfer Capability and Bifurcation Phenomena of Loosely Coupled Inductive Power Transfer Systems," IEEE Trans. Ind. Electron., vol. 51, no. 1, pp. 148-157, Feb. 2004.

[2] R. Bosshard, J. Mühlethaler, J. W. Kolar, and I. Stevanovic, "Optimized Magnetic Design for Inductive Power Transfer Coils," in Proceedings of IEEE APEC 2013 Conference, Long Beach, USA, pp. 1812-1819, Mar. 2013.

[3] Fei Yang Lin, Grant A. Covic, and John T. Boys, "Evaluation of Magnetic Pad Sizes and Topologies for Electric Vehicle Charging," IEEE Trans. Power Electron., vol. 30, no. 11, pp. 6391-6407, Nov. 2015.

[4] Lan Jianyu, Tang Houjun and Gen Xin, "Frequency Splitting Analysis of Wireless Power Transfer System Based on T-type transformer Model," Elektronika IR Elektrotechnika, vol. 19, no. 10, pp. 109-113, 2013.

[5] Sarp G. Cimen and Benedikt Schmuelling "Frequency Bifurcation Study of an Inductive Power Transmission System," in proceedings of IEEE POWERENG2013 Conference, Istanbul, Turkey, pp. 1185-1189, May. 2013.

[6] R. Bosshard, U. Badstübner, J. W. Kolar and I. Stevanovic, "Comparative Evaluation of Control Methods for Inductive Power Transfer," in proceedings of IEEE ICRERA2012 Conference, Nagasaki, Japan, pp.1-6, Nov. 2012.

[7] R. L. Steigerwald, "A comparison of half-bridge resonant converter topologies," IEEE Trans. Power Electron., vol. 3, no. 2, pp. 174-182, Apr. 1988.

[8] SangCheol Moon, Bong-Chul Kim, Shin-Young Cho, Chi-Hyung Ahn, and Gun-Woo Moon, "Analysis and Design of a Wireless Power Transfer System With an
Intermediate Coil for High Efficiency," IEEE Trans. Ind. Electron., vol. 61, no. 11, pp. 5861-5870, Nov. 2014.

[9] Steven Strogatz, "Non-linear Dynamics and Chaos: With Applications to Physics, Biology, Chemistry and Engineering," in Perseus Books, vol. 1, 2000, p.55-56.

[10] Wang-Qiang Niu, Jian-Xin Chu, Wei Gu, and ADDi Shen, "Exact Analysis of Frequency Splitting Phenomena of Contactless Power Transfer Systems," IEEE Trans. Circuits Syst., vol. 60, no. 6, pp. 16701677, Jun. 2013.

[11] J. A. Ferreira, "Electromagnetic Modelling of Power Electronic Converters," in Kluwer Academics Publishers, 1989.

[12] Jonas Mühlethaler, "Modeling and multi-objective optimization of inductive power components," Ph.D. dissertation, Swiss Federal Inst. Technol. Zurich, ETHZ, Zürich, The Switzerland, 2012.

[13] Lotfi. A. W, and Lee, F. C, "Proximity losses in short coils of circular cylindrical windings," in Proceedings of IEEE PESC 1992 Conference, Toledo, Spain, pp. 1253-1260, Jun. 1992.

[14] N. Guan, C. Kamidaki, T. Shinmoto and K. Yashiro, "AC Resistance of Copper Clad Aluminum Wires," in Proceedings of ISAP 2012 Conference, Nagoya, Japan, pp. 447-450, Oct. 2012.

[15] Reinert. J, Brockmeyer. A, and De Doncker.Rik.W.A. A, "Calculation of Losses in Ferro- and Ferrimagnetic Materials Based on the Modified Steinmetz Equation," IEEE Trans. Ind. Appl., vol. 37, no. 4, pp. 1055-1061, Jul/Aug. 2001.

[16] Yuji Sezai, "Ferrite Materials for Power Transformer PC95," Dempa Shimbun High-Technology, TDK Corporation, Jul. 2003.

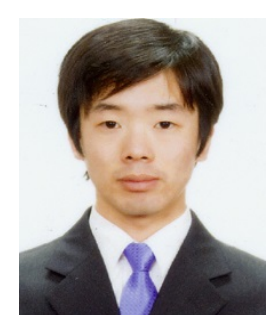

Minkook Kim $\mathrm{He}$ received the B.S degree in electrical engineering from Sungkyunkwan University, Suwon, Korea, in 2012, where he is currently working toward the combined M.S. and $\mathrm{Ph} . \mathrm{D}$. degree in electrical engineering. His research interest includes wireless power transfer system for

electric vehicles.

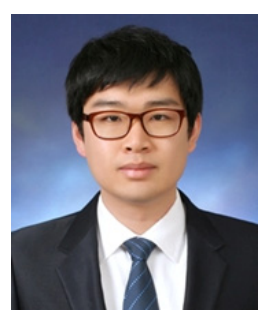

Jae-Woo Lee He received the B.S and the M.S. degrees in electrical and computer engineering from Sungkyunkwan University, Suwon, Korea, in 2014 and 2016, respectively. He joined the Living \& Energy R\&D center at LG Electronics, Seoul, Korea 
in 2016. His research interests include inductive heating and wireless power transfer.

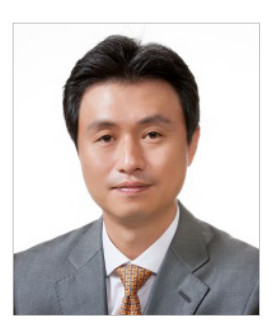

Byoung Kuk Lee He received the B.S. and the M.S. degrees from Hanyang University, Seoul, Korea, in 1994 and 1996, respectively and the Ph.D. degree from Texas A\&M University, College Station, TX, USA, in 2001, all in electrical engineering. From 2003 to 2005, he was a Senior Researcher with Power Electronics Group, Korea Electrotechnology Research Institute, Changwon, Korea. From 2006, he is with the College of Information and Communication Engineering, Sungkyunkwan University, Suwon, Korea. His research interests include on-board charger and wireless power transfer for electric vehicles, energy storage systems, hybrid renewable energy systems, dc distribution systems for home appliances, power conditioning systems for fuel cells and photovoltaic, modeling and simulation, and power electronics. Prof. Lee received the Outstanding Scientists of the 21st Century from IBC and listed on 2008 Ed. of Who's Who in America and 2009 Ed. of Who's Who in the World. $\mathrm{He}$ is an Associate Editor in the IEEE TRANSACTIONS ON INDUSTRIAL ELECTRONICS and Guest Associate Editor in the IEEE TRANSACTIONS ON POWER ELECTRONICS. He was the Presenter for Professional Education Seminar with the topic of "OnBoard Charger Technology for EVs and PHEVs" at the IEEE Applied Power Electronics Conference in 2014 and was the General Chair for the IEEE Vehicular Power and Propulsion Conference in 2012. 